\title{
Is the Faculty of Family Planning and Reproductive Health Care Guidance on emergency contraception being followed in general practice? An audit in the West Midlands, UK
}

\author{
Lisa Bannister, Joanna Macve, Benjamin Pinkey, Helen Webberley
}

\begin{abstract}
Background and methodology In 2003, the Faculty of Family Planning and Reproductive Health Care (FFPRHC) of the Royal College of Obstetricians and Gynaecologists published guidance on emergency contraception (EC). A literature search revealed no published work describing doctors' actions when prescribing EC. In order to assess the extent to which the FFPRHC Guidance is being followed in general practice, an audit of the medical notes of women requesting EC between January 2003 and December 2004 in six general practice surgeries located in the West Midlands, UK was conducted. From the medical notes, discussions between health care professionals and patients requesting EC regarding ongoing contraceptive needs, the risk of sexually transmitted infections (STIs) and the availability of the emergency intrauterine device (IUD) were recorded.
\end{abstract}

Results A total of 718 emergency contraceptive pill consultations were analysed. The median age for presentation was 24 years. The 20-24 years age group accounted for the most consultations (30.9\%). In $40 \%$ of consultations there was no evidence of future contraceptive needs having been discussed. Only 20 $(2.8 \%)$ consultation notes contained evidence that STIS had been discussed. Chlamydia tests were undertaken in only $15 / 718(1.7 \%)$ consultations. In only $10(1.4 \%)$ of the consultations was the IUD discussed with the patient as an alternative form of EC.

Discussion and conclusions This audit suggests that the FFPRHC Guidance on EC is not being followed in general practice, and therefore patients requesting $\mathrm{EC}$ may not be receiving the highest standard of care.

Keywords: audit, emergency contraception, FFPRHC Guidance, general practice, intrauterine device

J Fam Plann Reprod Health Care 2007; 33(3): 195-198

(Accepted 15 December 2006)

\section{Background}

Guidance from the Faculty of Family Planning and Reproductive Health Care (FFPRHC) of the Royal College of Obstetricians and Gynaecologists outlines the standard of care a woman should receive when she is prescribed emergency contraception (EC). Three of the recommendations are that women should be: (1) provided with information and counselling on the use of their contraceptive method of choice, (2) offered an intrauterine device (IUD) as an alternative to the emergency contraceptive pill and (3) offered sexually transmitted infection (STI) screening. ${ }^{1}$ The General Household Survey in 2002 reported that 7\% of women aged 16-49 years, living in Britain, who were not sterilised and whose partners were not sterilised, had used a method of EC in the last 12 months. ${ }^{2}$ The health of a large number of women is affected by the care they receive when they are prescribed EC and therefore it is important that general practitioners (GPs) consistently implement best clinical practice.

\section{Advice regarding ongoing contraceptive needs}

Women requesting EC from their GP have had unprotected sexual intercourse (UPSI) or contraceptive failure. The time when a woman presents to a GP requesting EC is an appropriate time to give advice and counselling about longterm contraception. These women may have had problems with their current contraceptive method or may not have been using any form of contraception. Counselling should include instruction and discussion of correct use of the

University of Birmingham Medical School, Birmingham, UK Lisa Bannister, MA, Medical Student

Joanna Macve, BSc, PhD, Medical Student

Benjamin Pinkey, MPharm, Medical Student

Helen Webberley, MBChB, MRCGP, General Practitioner and Lecturer

Correspondence to: Dr Helen Webberley, Neville Hall Hospital, Abergavenny NP7 7EG, UK. E-mail: helen@fort-royal.net

\section{Key message points}

- Requests for emergency contraception (EC) provide an ideal opportunity for health education concerning sexually transmitted infections.

- Patients requesting EC should be targeted for discussions around longer-term contraception.

- Patients attending for oral EC may not be being offered the more effective intrauterine method.

patient's current form of contraception or consideration of future contraceptive methods. ${ }^{1}$

The first aim of this study was to establish whether patients who attend their general practice surgery for EC are offered advice about, or supplied with, long-term contraception.

\section{Types of EC}

Two methods of EC are currently recommended in the FFPRHC Guidance: progestogen-only EC (POEC, available on prescription as Levonelle- $2^{\circledR}$ ) and copper intrauterine devices (IUDs). Clinical practice and efficacy have been described thoroughly. ${ }^{1}$ In the 2002 General Household Survey, $7 \%$ of all women aged 16-49 years had used EC in the last 12 months; of these only $3 \%$ had used an IUD, the majority used POEC. ${ }^{2}$

POEC is indicated within 72 hours of the first episode of UPSI or potential contraceptive failure. Overall, POEC prevents $84 \%$ of expected pregnancies within this time frame. ${ }^{3}$

A copper IUD can be inserted up to 5 days (120 hours) after the first episode of UPSI at any time in the menstrual cycle or up to 5 days after the expected date of ovulation in a regular cycle. ${ }^{1}$ Copper IUD devices are the most effective method of EC. They prevent over $99 \%$ of expected pregnancies $^{1}$ and are indicated when maximum effectiveness is a priority. The option of an IUD with its low failure rate and its potential for use as an ongoing 
Table 1 Age range of women prescribed emergency contraception

\begin{tabular}{lllllll}
\hline \multicolumn{7}{c}{ Age range (years) } \\
\cline { 2 - 7 } & $<\mathbf{2 0}$ & $\mathbf{2 0 - 2 4}$ & $\mathbf{2 5 - 2 9}$ & $\mathbf{3 0 - 3 4}$ & $\mathbf{3 5 - 3 9}$ & $\mathbf{4 0 +}$ \\
\hline Patients $[n(\%)]$ & $141(19.6)$ & $222(30.9)$ & $136(22.7)$ & $101(14.1)$ & $64(8.9)$ & $27(3.8)$ \\
\hline
\end{tabular}

method of contraception should be discussed with all women even if they present within 72 hours of UPSI. ${ }^{1}$

The second aim of this study was to establish whether, in the general practice setting, patients who are prescribed POEC have been offered a choice between the emergency contraceptive pill and the emergency IUD.

\section{Screening for STIs}

According to FFPRHC Guidance, all those attending for EC should have a sexual history taken to assess risk of STI. ${ }^{1}$ Service providers should offer STI screening to all those attending for EC. 1

Genital Chlamydia trachomatis infection is the most common sexually transmitted bacterial infection in England. The infection is usually asymptomatic but it is associated with serious complications such as ectopic pregnancy, pelvic inflammatory disease and, ultimately, infertility; complications in men include epididymitis. ${ }^{4}$

The results of the first full year of screening in the National Chlamydia Screening Programme recorded high rates of chlamydia positivity in the targeted population of under-25-year olds; levels were $10.1 \%$ in women and $13.3 \%$ in men. 5 A study of those attending community family planning services in the south of England for EC reported that $88.3 \%$ were under 25 years old and over half were teenagers. ${ }^{6}$ This is similar to findings in other studies. ${ }^{7}$ As large numbers of those requesting EC are aged under 25 years and there is a high prevalence of chlamydia amongst this population, this is an excellent opportunity to screen these individuals. A 2002 study in a family planning clinic setting advocates that screening for chlamydia infection is indicated for women aged under 30 years using EC. It also demonstrates that screening at the time a woman attends for EC is adequate to detect the great majority of infected women. 8

The final aim of this study was to establish whether STI risk is assessed and chlamydia screening offered to patients requesting $\mathrm{EC}$ in general practice.

\section{Methods \\ Recruitment of practices}

Six general practices in the West Midlands were invited to take part in the audit. The practices were known to the student authors of this paper, having been allocated randomly to them during their clinical training at Birmingham Medical School. All six practices agreed to participate. Together, the practices covered a total patient population of around 45000 . The practices were located in urban centres in the West Midlands. The practice sizes ranged from approximately 4000 to 11000 patients.

\section{Identification of subjects}

The computer database in each practice was searched for all patients who had been prescribed Levonelle- 2 between 1 January 2003 and 31 December 2004. The computer record for each resultant consultation was examined. In practices where there was no computer record for that consultation, paper records were studied.

\section{Measures and procedure}

A proforma was used by the three auditors to ensure consistency. Details of the prescribing doctor or nurse, the patient's age and the year in which the consultation took place were recorded. If more than one consultation for a particular patient had occurred within the study period, details of each consultation were recorded separately.

For each consultation where POEC was prescribed it was recorded whether there was evidence of the following in the notes: the time since UPSI, whether chlamydia testing had occurred, whether there had been discussion about STIs, whether there had been discussion about use of an IUD as an alternative to POEC, and whether ongoing contraceptive needs had been discussed.

The data were analysed using Excel $^{\mathrm{TM}}$.

\section{Ethical approval}

The study was approved by the clinical governance and audit manager for South Birmingham Primary Care Trust.

\section{Results}

A total of 537 patients were prescribed Levonelle-2 between 1 January 2003 and 31 December 2004. The total number of consultations in the study period was 718 since some women were prescribed POEC on more than one occasion within the study period. The majority (435, $81.0 \%$ ) of the women had only one consultation and $102(19.0 \%)$ women had more than one consultation. The highest number of prescriptions for a single patient in the 2-year study period was 11 .

GPs conducted 574/718 (79.9\%) consultations for EC. Of the remaining consultations, $106(14.8 \%)$ were with practice nurses. It was not possible to ascertain which health professional had been involved in 38 (5.3\%) of the consultations.

The median age for presentation was 24 (range, 14-51) years. Of 718 prescriptions, approximately $50 \%$ were made to women aged under 25 years (Table 1 ).

In $237(33.0 \%)$ consultations, the time that had elapsed between UPSI and presentation was not recorded. Of the 481 consultations where the time was recorded, $59.0 \%$ of women presented within 24 hours of intercourse (Figure 1). Less than $1 \%$ of women presented more than 72 hours after intercourse had taken place.

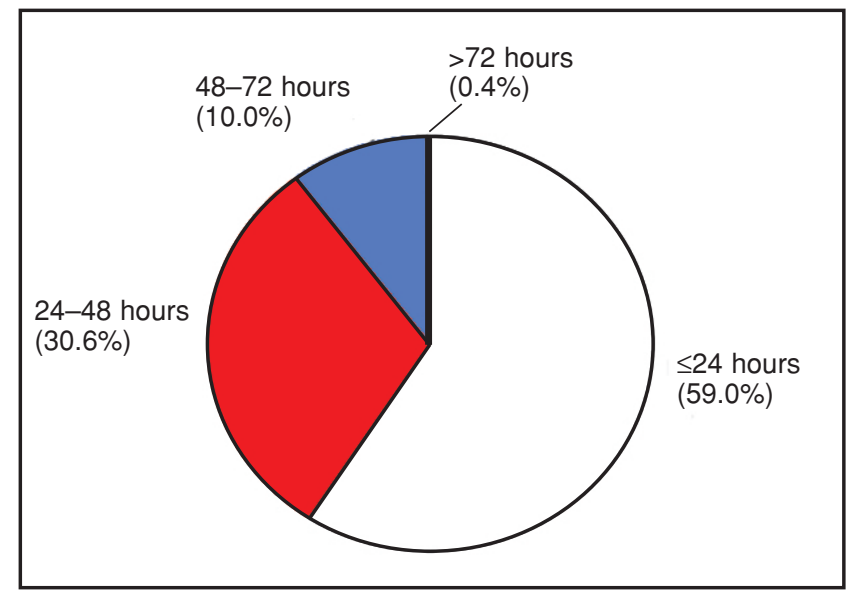

Figure 1 Time between unprotected sexual intercourse and consultation for prescription of emergency contraception 


\section{Advice regarding ongoing contraceptive needs}

There was no record of information and counselling regarding ongoing contraception being given in 286/718 $(39.8 \%)$ consultations. In 432 consultations long-term contraception was discussed. Overall, $12.4 \%$ of women were using condoms as their first choice of contraception, 9.9\% were taking the oral contraceptive pill, $3.1 \%$ were given condoms in the course of the consultation and $17.7 \%$ were prescribed the oral contraceptive pill in addition to POEC. In the remaining $17.1 \%$ of consultations discussion regarding long-term contraception was noted, although there was no information regarding current or future contraceptive provision.

\section{Types of EC}

Discussion regarding the emergency IUD was recorded in only $10 / 718(1.4 \%)$ consultations. In two of these consultations the fitting of an emergency IUD was arranged. In nine of the consultations where discussion of the emergency IUD was noted, the time since UPSI was recorded as follows: six women presented within 24 hours; one woman presented within 48 hours and two women were seen within 72 hours.

\section{STI discussion and chlamydia testing}

Discussion regarding STIs was recorded as having taken place in only $20 / 718(2.8 \%)$ consultations. Only $15 / 718$ $(1.7 \%)$ women were tested for chlamydia. The majority of STI discussions $(13 / 20,65 \%)$ and chlamydia testing $(10 / 15,66.7 \%)$ occurred in consultations with patients aged 20-30 years.

\section{Discussion}

This audit of medical records has allowed assessment of the quality of care provided to women seeking EC in a large number of consultations. Use of an audit negated the need to identify and question patients seeking EC in order to gain information about this potentially sensitive issue. One inevitable limitation of the present study is that the results are dependent on how thoroughly consultations were documented in general practice.

\section{Users}

Women aged 20-24 years were the most frequent attendees for POEC over the 2-year period studied (2003-2004), accounting for $30.9 \%$ of all prescriptions. Approximately half of all users were aged under 25 years and almost $75 \%$ were under 30 years old. These findings are similar to those described by the 2002 General Household Survey, which reported EC was most likely to have been used by women under 25 years, and that those aged under 25 years were twice as likely to have used EC than those aged over 30 years. ${ }^{2}$

\section{Time since UPSI}

In order for the health practitioner to assess the risk of pregnancy, FFPRHC Guidance recommends that an accurate sexual history be taken; this should include an enquiry as to when the first episode of UPSI took place. ${ }^{1}$ This will allow the health care professional to give advice regarding the most appropriate method of EC and make appropriate follow-up arrangements. In the present study, the time that had elapsed since UPSI was not recorded in $33.0 \%$ of consultations.

Of the 481 consultations where the time was recorded, $59.0 \%$ took place within 24 hours of UPSI. Two previous studies, in family planning clinics, have recorded that lower proportions of women $(44.8 \%, 41 \%)$ presented within 24 hours of UPSI. 6,7 The higher percentage in this study may reflect differences between users of, or access to, the different services.

\section{Advice regarding ongoing contraceptive needs}

In $40 \%$ of all consultations there was no record of information and counselling regarding ongoing contraception being given to the patient. This result is surprising considering that the consultations were necessitated by a failure of contraception. This contrasts with a recent telephone interview study of GPs' prescribing habits of EC in which it was reported that $77.6 \%$ of GPs said they would discuss future contraception with patients. 9

There could be a number of explanations as to why doctors are failing to meet this criterion. Doctors work within appointment schedules, meaning that their time with any one patient is restricted. A doctor may prefer to deal with only the presenting complaint at the initial consultation. Additionally, the doctor may feel that it is not appropriate to discuss long-term contraception if a woman is distressed by her current situation. The fact that almost $20 \%$ of the study population required EC more than once within the study period might suggest that women are not being seen subsequently about their contraceptive needs if the doctor chooses to delay this discussion. Dealing with the issue of long-term contraception immediately may in fact save time in the future and prevent women returning with the same problem at a later date.

Our results show that at presentation for EC $22 \%$ of women were already using long-term contraception. A survey by the Office of National Statistics reports that women currently using contraception accounted for seveneighths of women who had used the 'morning-after pill' at least once in the last year. ${ }^{10}$ Other studies have also reported much higher numbers of women using some form of contraception before requesting EC than we have found in the present study.4,9 This discrepancy between our results and those of other studies may be due to the doctors in our practices not accurately recording in the patients' notes what has happened in the consultation (i.e. if the woman was using contraception and it was not documented).

In $21 \%$ of consultations the doctor provided the patient with contraception either in the form of the oral contraceptive pill $(18 \%)$ or condoms $(3 \%)$. In a further $17 \%$ of consultations long-term contraception was discussed but no actions were taken. In these consultations the FFPRHC Guidance had been adhered to.

\section{Types of EC}

Only in 10/718 consultations was it recorded that the emergency IUD had been discussed. Therefore, it appears patients are rarely being given a choice between POEC and the emergency IUD. There may be a number of reasons why doctors are not offering women the emergency IUD. A study that examined the barriers to the use of IUDs as EC found that GPs perceive the provision of emergency IUDs as expensive in terms of time and effort. ${ }^{11}$

Doctors may not be trained in the fitting of IUDs; and even if they are competent at the procedure, it is much easier to give POEC than fit or counsel and refer for IUD insertion. Fears amongst health care professionals regarding the safety of IUDs may also result in a reluctance to offer this service to patients.

\section{STI screening and discussion}

Of 718 consultations in which Levonelle-2 was prescribed only $20(2.8 \%)$ resulted in a recorded discussion about STIs. As stated in the FFPRHC Guidance, ${ }^{1}$ discussion regarding STIs is important in this group of women; 
presentation for EC implies that barrier contraception has not been used or has failed. There may be a number of explanations why doctors are not discussing STIs with their patients. One reason could be that they feel the time constraints of the consultation do not allow them to explore these issues. Doctors may feel uncomfortable having a discussion about the patient's sexual activity. Alternatively, doctors may not be aware that STI discussion and sexual history taking are a part of current EC guidance. The previous FFPRHC Guidance written in 2000 did not cover this area. 12

Samples to test for chlamydia infection were taken in only $15(2.1 \%)$ consultations despite recommendations to offer STI screening to all patients attending for EC. ${ }^{1} \mathrm{~A}$ recent study of barriers to opportunistic chlamydia testing outlined a number of reasons why screening was not occurring in general practice. These explanations included: lack of knowledge of the benefits of testing and when and how to take specimens, lack of time, worries about discussing sexual health, and lack of guidance. Health care staff stated that any increased testing should be accompanied by clear, concise Primary Care Trust guidance on when and how to test, including how to obtain informed consent and perform contact tracing. ${ }^{13}$ It could be, however, that doctors are offering screening but patients are refusing it and the doctor's offer has not been recorded in the notes.

A study looking into the clinical management of chlamydia supports our finding that few tests for chlamydia are being carried out in general practice. In that study, $42 \%$ of GPs reported carrying out only between one and four tests a month and $35 \%$ of GPs reported performing less than one test a month. ${ }^{14}$

\section{Conclusions}

Requests for EC provide a valuable opportunity for discussion around better prevention of unintended pregnancy and STIs. General practices are failing to adhere to FFPRHC Guidance on sexual history taking, future contraceptive needs and the use of the IUD as a method of EC. It may be possible to improve adherence to the Guidance by relatively simple measures such as the use of protocol proformas to guide consultations, the booking of double appointments for those requesting EC to allow time for more meaningful consultations, further training in this area for both doctors and nurses, and an increase in the role of practice nurses in this area of health provision.

In order to combat the rising rates of genital chlamydia infection and the high rates of unplanned pregnancies in the UK, now more than ever it is important that clinicians are implementing best practice in every consultation for EC.
We hope that the publication in April 2006 of new FFPRHC Guidance on EC will increase awareness about this issue amongst health professionals.

\section{Acknowledgements}

The authors would like to thank the partners at the six medical practices for allowing them to audit their practices' medical records. They are also grateful to Anne Webb for her helpful comments.

Statements on funding and competing interests

Funding None identified.

Competing interests None identified.

\section{References}

1 Clinical Effectiveness Unit of the Faculty of Family Planning and Reproductive Health Care (FFPRHC). FFPRHC Guidance on emergency contraception (April 2003). J Fam Plann Reprod Health Care 2003; 29(2): 9-16.

2 Rickards L, Fox K, Roberts C, Fletcher L, Goddard E. Living in Britain. Results from the 2002 General Household Survey (Chapter 10: Contraception; 175-190). 2004. http://www.statistics.gov.uk/lib2002/downloads/contraception. pdf [Accessed 11 November 2006].

3 von Hertzen H, Piaggio G, Ding J, Chen J, Song S, Bartfai G, et al; WHO Research Group on Post-ovulatory Methods of Fertility Regulation. Low dose mifepristone and two regimens of levonorgestrel for emergency contraception: a WHO multicentre randomised trial. Lancet 2002; 360: 1803-1810.

4 Moens V, Baruch G, Fearon P. Opportunistic screening for Chlamydia at a community based contraceptive service for young people. BMJ 2003; 326: 1252-1255.

5 LaMontagne DS, Fenton KA, Randall S, Anderson S, Carter P. Establishing the National Chlamydia Screening Programme in England: results from the first full year of screening. Sex Transm Infect 2004; 80: 335-341.

6 Shawe J, Ineichen B, Lawrenson R. Emergency contraception: who are the users? J Fam Plann Reprod Health Care 2001; 27: 209-212.

7 Roizen J, Garside R, Barnett L. Repeat use of emergency contraception: how frequent is it? J Fam Plann Reprod Health Care 2001; 27: 197-201.

8 Kettle H, Cay S, Brown A, Glasier A. Screening for Chlamydia trachomatis infection is indicated for women under 30 using emergency contraception. Contraception 2002; 66: 251-253.

9 Ziebland S, Graham A, McPherson A. Concerns and cautions about prescribing and deregulating emergency contraception: a qualitative study of GPs using telephone interviews. Fam Pract 1998; 15: 449-456.

10 Dawe F, Rainford L. Contraception and Sexual Health, 2003. 2004. http://www.dh.gov.uk/assetRoot/04/08/99/46/04089946. pdf [Accessed 11 November 2006].

11 Reuter S. Barriers to the use of IUDs as emergency contraception. Br J Fam Plann 1999; 25: 63-68.

12 Faculty of Family Planning and Reproductive Health Care. Guidance April 2000. Emergency contraception: recommendations for clinical practice. Br J Fam Plann 2000; 26: 93-96.

13 Ziebland S. Emergency contraception: an anomalous position in the family planning repertoire? Soc Sci Med 1999; 49: 1409-1417.

14 Griffiths C, Cuddigan A. Clinical management of chlamydia in general practice: a survey of reported practice. J Fam Plann Reprod Health Care 2002; 28: 149-152.

\section{BOOK REVIEW}

Memories After Abortion. V Wahlberg (ed.). Oxford, UK: Radcliffe Publishing Ltd, 2006. ISBN: 1-84619-131-9. Price: £17.95. Pages: 111 (paperback)

This helpful book provides a wide perspective on young people's experience of abortion.

The editor has spent a working lifetime in reproductive and sexual health care in Sweden and writes with authority and compassion. Although clearly rooted in Swedish experience, this book has much to say to a wider audience. A useful section sketches the historical context of liberalisation of abortion in Sweden and allows interesting comparisons with abortion legislation in other countries. Elsewhere, there are specific comparisons of attitudes to abortion in Italy and Sweden, acknowledging the vast historical differences but predicting increasing uniformity across Europe.

A particularly interesting chapter focuses on the experiences of young men involved in unwanted pregnancy. Theirs is a voice seldom heard. This chapter also reports extensive research on the risk behaviours and health needs of these young men as a group.

There is also a useful reflection on abortion from various ethical perspectives. This is refreshing and challenging reading at a time when ethical discussions can be polarised and dangerously simplified.

Throughout this book, quotes from young people experiencing abortion keep the discussion fresh and pertinent. Each chapter is also well referenced.

This book would be illuminating for any professionals involved in reproductive and sexual health or the care of young people.

Reviewed by Kate Weaver, MBChB, MFFP Staff Grade Doctor in Reproductive Health Care, Edinburgh, UK 